\title{
Understanding Election Results with Different Types of Maps
}

\author{
Spörl, Kristina ${ }^{\mathrm{a}}$, Wilkening, Jan ${ }^{\mathrm{b}} *$, Kirschenbauer, Sabine ${ }^{\mathrm{c}}$ \\ ${ }^{a}$ Kristina Spörl, Landeshauptstadt München e-mail: kristina.spoerl@muenchen.de \\ ${ }^{b}$ Jan Wilkening, University of Applied Sciences Würzburg-Schweinfurt e-mail: jan.wilkening@fhws.de * \\ ${ }^{c}$ Sabine Kirschenbauer, University of Applied Sciences München e-mail: sabine.kirschenbauer@hm.edu \\ * Corresponding author
}

Keywords: Geovisualization, Thematic Maps, Analysis of Election Results, User Studies

\begin{abstract}
:
Analyzing election patterns from a spatial view, interesting geographical patterns emerge. For instance, there often is a huge difference between voting shares for certain parties between urban and rural areas. One recurrent phenomenon in Europe and other places is that Green parties are stronger in urban areas, while in rural areas the conservative parties are ahead.
\end{abstract}

However, it goes without saying that there is a more complex pattern that needs to be analyzed. Often, demographic and socio-economic variables like religion, purchasing power, education or unemployment rate, which differ between certain regions, explain the differences in voting shares more precisely than classical rural-urban dichotomies. In this study, we focus on the following questions: How can such patterns be analyzed and visualized with interactive cartography? How do people perceive and interpret such patterns? How can cartography help in communicating such complex relationships effectively and efficiently?

While maps about voting shares are ubiquitous after every election, there are less examples of geovisualizations that show the statistical relationship between certain explanatory variables and voting shares (e.g. Lysek et al. 2020, Mansley \& Demšar 2015, Skoutaris 2017). Maps that depict the spatial disparities in these statistical relationships are even harder to find. Creating a geovisualization for this purpose is a challenging task: The choice of appropriate methods of thematic cartography and the collection and aggregation of statistical data is sophisticated itself. Adding the complex aspect of statistical significance to these geovisualizations makes it even more demanding.

In this study, we collected and aggregated data about parliamentary elections in Germany and socio-demographic data from different sources (e.g., the German National Election Office and the geomarketing company Nexiga GmbH). The spatial level of our analysis are the 299 electoral districts, which contained 155,149 voters on average. We focused on the voting shares for the six strongest parties.

After aggregating data in Excel and creating choropleth maps for the voting shares of the six strongest parties in ArcGIS Pro, we analyzed the statistical significance of several explanatory variables. Our explanatory variables contained population density, shares of Catholics and Protestants, balance of migration, purchasing power, unemployment rate, young voters (18-29 years), elderly voters (>60 years), share of households with low income, share of people with university degree, foreigner ratio, and car ownership.

We then created a multiple regression model with the Ordinary Least Squares (OLS) tool of the Spatial Statistics toolbox. This tool also provides a statistical output in text form that can be used to see which explanatory variables are statistically significant (p-value and similar statistic indexes). For instance, we could identify a strong relationship between the explanatory variables of share of Catholics, purchasing power (both positive), unemployment rate and share of young voters (both negative) with the voting share for the conservative party. For the voting share of the Green party, we could identify statistically significant relationships with the number of academic degree holders, foreigner ratio (both positive), car ownership and unemployment rate (both negative).

The next step was creating various choropleth maps in ArcGIS Pro and sharing them as web maps on ArcGIS Online. These web maps were then integrated in ArcGIS StoryMaps and enhanced with explanatory text and additional graphics. We created two web-based "stories", that is, two geovisualizations (see Figure 1): One complex ArcGIS StoryMap where information about voting shares for a party and several explanatory variables were shown in one map (complex map), and another storymap where the similar information can be found in small multiples (analytical map). 

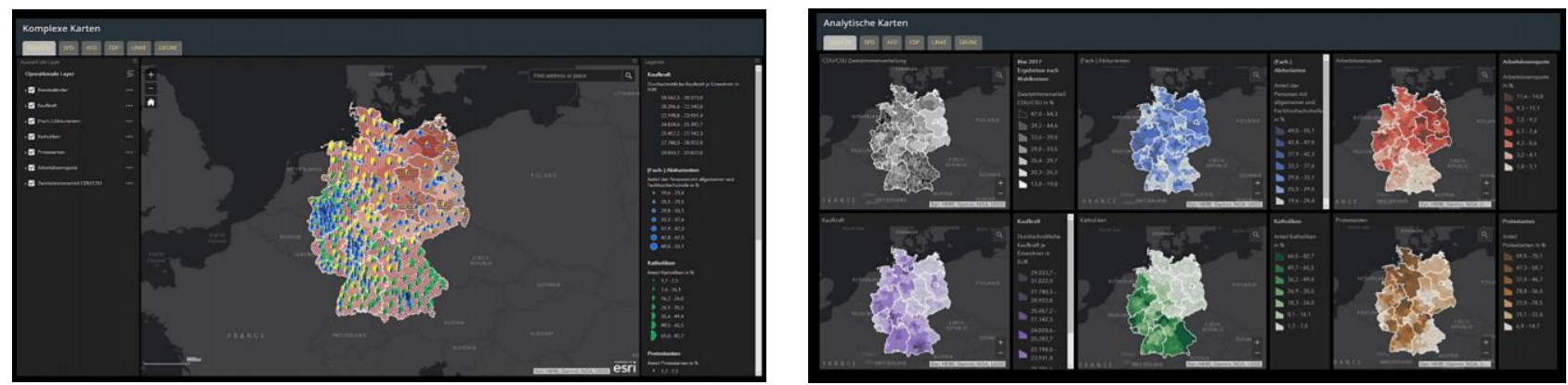

Figure 1. Complex map (left) and analytical maps (right) of election results.

Based on these StoryMaps, we then conducted an empirical user study of "between-subject design" to investigate which type and method of visualization helps users better in understanding statistical relationships between independent and dependent variables. The study was carried out as a web-based experiment with 24 users. Some of them work in the cartography and GIS industry, while others deal with maps only through the media.

In the user study, participants were asked several questions about the relationship of explanatory variables and voting shares, which could all be answered by interacting with the StoryMaps. In the first group of tasks, participants were asked to identify whether a relationship between explanatory variables and voting shares was positive, negative, or non-existent. In the second group of tasks, they should identify electoral districts which fulfil certain criteria.

Our results suggest that users generally understand statistical relationships easier with analytical maps (small multiples) than with complex maps, where all pieces of information are shown in only one visualization. However, these results depend on the task: In the second group of tasks (identifying electoral districts), the complex maps performed better than in the first group of tasks (analyzing statistical relationships).

It has also become apparent that, depending on the goal of the visualization and the target group of the geovisualization, different types of maps are better suitable to communicate certain facts. For instance, our results suggest that analytical maps (small multiples) are more suitable for the presentation of statistical relationships, while more extensive tasks can be solved better with complex maps.

Further research is needed to establish the results of the study and to identify aspects of map design that help people to better understand voting results and the geographical pattern behind these results. With open data initiatives emerging, more and more election and demographic data is publicly available all over the world. Moreover, GIS tools are becoming more powerful in analyzing and visualizing statistical data with a geo-spatial component. The integration of open-source libraries in R and Python like ggplot2, seaborn or matplotlib provide further opportunities for such analyses. Enhancing similar results about the accuracy of answers with eye-tracking analyses provides even more possibilities. Another opportunity for geovisualization is using the third dimension for additional variables.

It remains to be seen whether our patterns - not only the patterns of statistical significance, but also the patterns of comparing and evaluating map types - can be replicated with results of further elections with similar election systems all over the world. Further analysis is also needed to explore which methods of data visualization and thematic cartography suit users of "election- results-maps" best, especially under consideration of our today's fast-paced society.

\section{References}

Lysek, J., J. Pánek \& T. Lebeda (2020): Who are the voters and where are they? Using spatial statistics to analyse voting patterns in the parliamentary elections of the Czech Republic. Journal of Maps 17, 2021.

Mansley, E. \& U. Demšar (2015): Space matters: Geographic variability of electoral turnout determinants in the 2012 London mayoral election. Electoral Studies , 40, 322-334.

Skoutaris, C. (2017): Modeling Election Results as a Function of Geodemographical and Lifestyle Variables. Master of Science Thesis in Geoinformatics School of Architecture and the Built Environment Royal Institute of Technology (KTH) Stockholm, Sweden. 